\title{
Vulvar varicosities: diagnosis, treatment, and prevention
}

\author{
This article was published in the following Dove Press journal: \\ International Journal of Women's Health \\ 28 June 2017 \\ Number of times this article has been viewed
}

\section{Sergey G Gavrilov}

Faculty of Medicine, Pirogov Russian National Research Medical University, Moscow, Russia
Correspondence: Sergey G Gavrilov Faculty of Surgery and Urology, Pirogov Russian National Research Medical University, I Ulitsa Ostrovitianov,

Moscow I I7997, Russia

$\mathrm{Tel}+79169299947$

Fax +7 4956338695

Email gavriloffsg@mail.ru
Objective: Vulvar varicosity is a relatively common venous disorder in women with varicose veins of the pelvis and lower extremities and in pregnant women, but there is little information in the medical literature concerning its diagnosis and management. The aim of this study was to describe our experience with 101 women with vulvar varicosities who were examined and treated at our center during 2000-2014.

Patients and methods: Patients were divided into two groups: group 1 with 61 women with varicose veins of the pelvis and enlarged vulvar veins, and group 2 with 40 pregnant women (11-38 weeks of pregnancy) with vulvar varicosities.

Results: In most cases, vulvar varicosities were able to be diagnosed at clinical examination. In both groups, duplex ultrasound of the veins of the pelvis and lower extremities was used to confirm the presence of varicose veins, identify any retrograde flow, accurately determine vein diameter, and to reveal any anatomical feature of the intrapelvic veins. Treatment approaches varied significantly between the two groups from purely conservative measures during pregnancy to surgical procedures on the ovarian and vulvar veins in group 1 . The venoactive agent, micronized purified flavonoid fraction, was effective at reducing the symptoms of vulvar varicosities in both groups.

Conclusion: A tailored approach to the diagnosis and treatment of vulvar varicosities can significantly improve the quality of care of women with this disorder.

Keywords: micronized purified flavonoid fraction, pelvic varicose veins, pelvic venous congestion, vulvar varicosities

\section{Introduction}

Vulvar varicosities, or varicose veins of the vulva, ${ }^{1}$ are dilated veins in the labia majora and labia minora, and are estimated to occur in $22 \%-34 \%$ of women with varicose veins of the pelvis and in $18 \%-22 \%$ of pregnant women. ${ }^{2}$ During the postpartum period, perineal veins may persist and enlarge with time in $4 \%-8 \%$ of patients. ${ }^{2,3}$ Vulvar varicosities are associated with venous thromboembolic events, both during pregnancy and in the nonpregnant state, superficial dyspareunia, and vulvodynia. They may also cause psychoemotional and family problems. It is difficult to estimate reliably the prevalence of this pathological condition, as vulvar varicosities often remain undiagnosed because of the atypical localization of the varicose veins, women's reluctance to consult, and in some cases the absence of any discomfort.

The anatomical basis for the development of vulvar varicosities relates to the connections between the veins of the pelvis and external genitals. ${ }^{4}$ Vulvar veins drain into the external and internal pudendal veins, which deliver blood to the great saphenous vein and internal iliac vein. The veins of the labia majora and labia minora anastomose with the uterovaginal plexus. In addition, the connection to the pelvic veins is provided 
via the obturator vein and superficial circumflex iliac vein, as well as the groin, clitoral, and perineal perforant veins.

It is clear from a review of these anatomical connections that surgery on ovarian veins (resection, embolization) will have no effect on the dilated vulvar veins. Only a reduction in blood flow in the tributaries of the internal iliac vein will lead to the elimination of pelviperineal venous reflux and the disappearance of vulvar varicosity. There is a paucity of information in the medical literature on the diagnosis and management of vulvar varicosities. The aim of this prospective study was to describe our observations of this condition and to develop a diagnostic and therapeutic approach to vulvar varicosities in various clinical settings.

\section{Materials and methods}

A total of 101 women with vulvar varicosities (mean age 29.6 \pm 4.3 years) were examined and treated at the Clinic of Departmental Surgery, Pirogov Russian National Research Medical University during 2000-2014. The total follow-up period was 8 years (mean $5.6 \pm 1.2$ years). Patients were divided into two groups: group 1 with 61 women with varicose veins of the pelvis and enlarged vulvar veins, and group 2 with 40 pregnant women (11-38 weeks of pregnancy) with vulvar varicosities. The scope of examination, as well as treatment approaches and methods, varied significantly between the two groups.

The study was approved by the ethics committee of Pirogov. All patients provided written consent for the publication of their images and records. Statistical analyses were performed using Statistica 6.0 software, and included mean values and tests of significance.

\section{Group I}

In addition to a clinical and gynecological examination, all patients underwent duplex ultrasound of the veins of the pelvis and lower extremities. Ultrasonography is indicated not only to confirm the presence of varicose veins but also to identify any retrograde flow, accurately determine vein diameter, and reveal any anatomical features of the intrapelvic veins. It was used to examine the veins of the labia majora, estuarine tributaries of the great saphenous vein and its trunk, and connections between these vessels. Pelvic veins examined included the vaginal, uterine, parametrial, ovarian, iliac, and renal veins and inferior vena cava. Pain was measured using a modified pain scale that allows evaluation of an individual's tolerance to pain (Figure 1).

Further examination of patients was dependent on the results of the duplex ultrasound. In the event of enlargement or valvular incompetence of ovarian veins, signs of pelvic venous congestion (eg, chronic pelvic pain, dyspareunia, dysmenorrhea $)^{5}$ or scheduled surgery on the ovarian veins, patients underwent multislice computed tomography (MSCT) or selective ovariography with pelvic phlebography (SOPP) to verify the diagnosis, clarify the anatomical features of the pelvic veins, assess the state of the internal iliac vein tributaries, and to exclude or verify the mesoaortic compression of the left renal vein or left common iliac vein. Such studies were performed in 17 patients with painful varicose veins of the pelvis. In order to assess changes in venous outflow from the pelvic organs both before and after phlebectomy in the perineum, emission $\mathrm{CT}$ of the pelvic veins using in vivo labeled red blood cells was performed in 24 patients with varicose veins of the pelvis.

\section{Group 2}

The opportunities for examination and treatment of pregnant women with vulvar varicosities are limited. All patients were referred for consultation with an obstetrician-gynecologist for further diagnosis and advice on antenatal management of vulvar varicosities. In most cases, the diagnosis was based on clinical data (complaints, medical history, and local status). Patients with severe pain in the perineum, hypogastrium, or suspected thrombotic lesions of the vulvar or intrapelvic

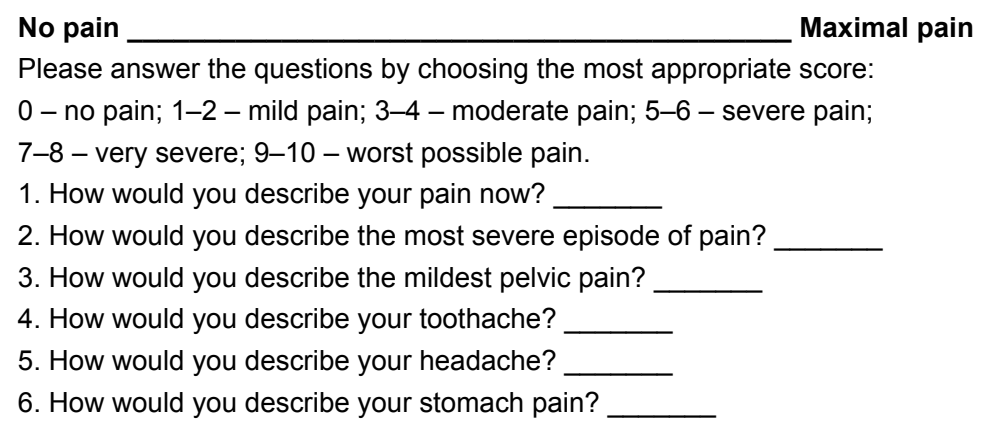

Figure I Modified pain-intensity and individual pain-tolerance scale. 


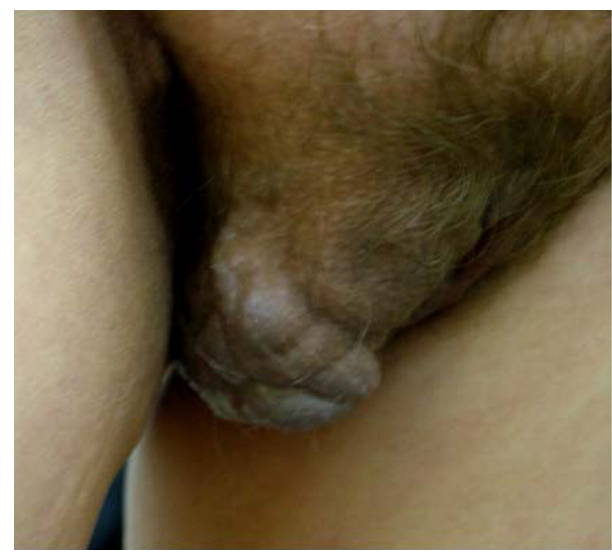

Figure 2 Appearance of vulvar varicose veins.

veins underwent duplex ultrasound examination of the veins of the perineum and pelvis.

\section{Results \\ Prevalence \\ Group I}

In the current study, vulvar varicosities were associated with varicose veins of the pelvis in 19 (32\%) cases; the remaining patients had asymptomatic pelvic venous congestion. Rarely, we observed patients with varicose veins of the round ligament of the uterus and the labia majora, who were wrongly diagnosed as having inguinal hernia and Bartholin gland cyst, respectively. Dilation of vulvar veins occurred most often in women with a history of two or more full-term pregnancies (91\%) and at a gestational age of 12-24 weeks (78\%). Subsequent pregnancies were accompanied by an earlier appearance and significantly greater dilation of the vulvar veins. Analysis of temporal parameters of vulvar varicosity occurrence by number of pregnancies and gestational age showed that during the first pregnancy, patients noted dilation of the vulvar veins at 18-24 weeks, and during the second pregnancy at 12-18 weeks. Vulvar varicosities were bilateral in $37.5 \%$, left-sided in $30 \%$, and right-sided in $32.5 \%$ of patients.

\section{Group 2}

In pregnant women, vulvar varicosities occurred most frequently at $18-20$ weeks' gestation. The pregnancy was their first for 27 women, second for six, and third for seven (only full-term pregnancies were taken into consideration). Among the 13 patients who had had more than one pregnancy, nine reported a relapse of vulvar varicosities at 12-14 weeks and four reported dilation of vulvar veins for the first time. In most patients $(77.5 \%)$ this was thus the first occurrence of vulvar

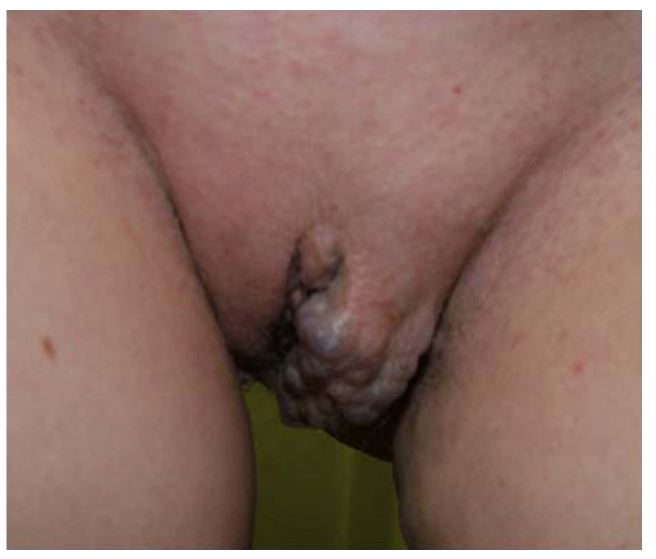

varicosities. Patients with consecutive pregnancies reported earlier occurrence. Dilation of veins was bilateral in $43.5 \%$ of women, left-sided in $27 \%$, and right-sided in $29.5 \%$. These figures were in general similar to group 1 .

\section{Clinical manifestations Group I}

The diagnosis of vulvar varicosities did not pose a significant challenge, and could be made by routine clinical examination (Figure 2). Clinical manifestations included findings of varicose veins on the labia majora or labia minora, pain in the vulvar area, heaviness and burning in the perineum, and swelling of the labia at the end of the day. The incidence rates for vulvar varicosity symptoms are presented in Figure 3. A steady progression and combination with varicose transformation of the intrapelvic veins was a characteristic feature of the clinical course of vulvar varicosities in group 1.

Varicose veins of the pelvis were associated with chronic pelvic pain in 19 (31\%) patients, coital or postcoital pain in $28(45 \%)$, hypogastric discomfort in $37(60 \%)$, menstrual disorders in eight (13\%), and frequent or painful urination in three (4.9\%) patients (Figure 4). Pelvic pain was registered in only a third of patients (31\%) with both vulvar varicosities

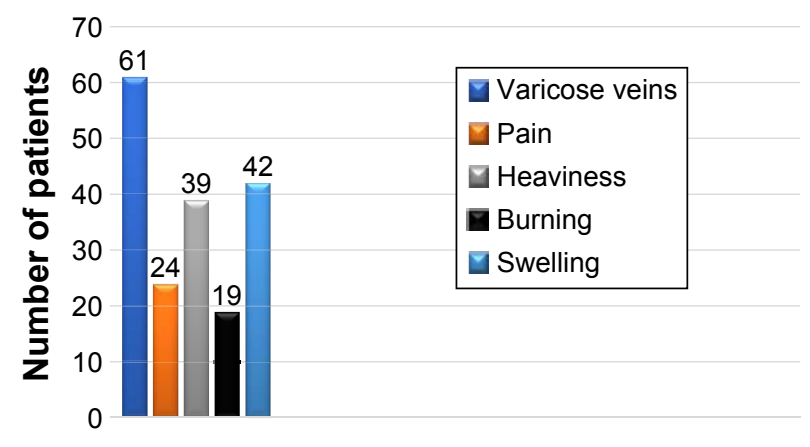

Figure 3 Clinical manifestations of vulvar varicosities. 


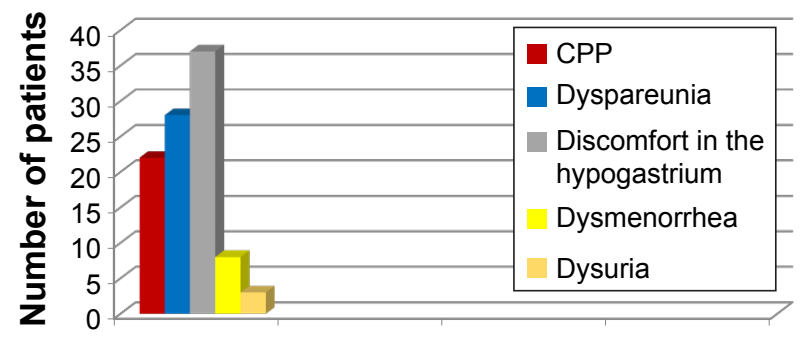

Figure 4 Symptoms of pelvic venous congestion in patients with vulvar varicosities and pelvic varicose veins.

Abbreviation: CPP, chronic pelvic pain.

and varicose veins of the pelvis compared to $70 \%$ of women with pelvic varicose veins alone. Discomfort in the lower abdomen was the most common symptom, occurring in most patients after static and exercise load.

\section{Group 2}

Clinical manifestations of vulvar varicosities in pregnant women were accompanied by complaints of varicose veins in the perineum, discomfort in this area, and swelling of the vulvar lips and perineum (Figure 5). In pregnant women, the symptoms associated with vulvar varicosities were more pronounced and characterized by the combination of vulvar, perineal, and inguinal varicosities with symptoms of hormone-induced phlebopathy (swelling, heaviness, fatigue, presence of telangiectasia, reticular veins). Varicose disease of the lower extremities was present in the vast majority of patients $(87 \%)$. The most common complaints were pruritus and skin maceration in the area of the vulvar varicose veins. No bleeding from vulvar veins or any indications of such a complication were observed. Two patients had symptoms of thrombophlebitis of the vulvar veins at 28 and 32 weeks of pregnancy, respectively, including intense pain in the area of the varicose veins, erythema, and perivascular edema (Figure 6).

Pelvic pain during pregnancy is quite common, and recurrent pain in the ilioinguinal and suprapubic region was thus not regarded as a sign of intrapelvic venous pathology. However, intense pain in the hypogastrium and hyperthermia requires (besides an obstetrician-gynecologist examination) transabdominal and transvaginal duplex ultrasound to exclude pelvic vein thrombosis.

\section{Instrumental diagnosis \\ Group I}

Duplex ultrasound revealed drainage of the veins of the labia majora into the dilated superficial external pudendal vein in three $(4.9 \%)$ patients, which was a contributing factor to the development of vulvar varicosities. A connection between the vulvar and vaginal veins was found in all patients. Ultrasonography also confirmed the presence of dilated veins in the uterus and parametrium in all cases (with a diameter of 5-11 mm). Reflux in these veins was identified in 54 patients. Dilated ovarian veins with retrograde blood flow were found in 23 patients, and dilated pudendal veins without retrograde flow in four patients. No abnormalities
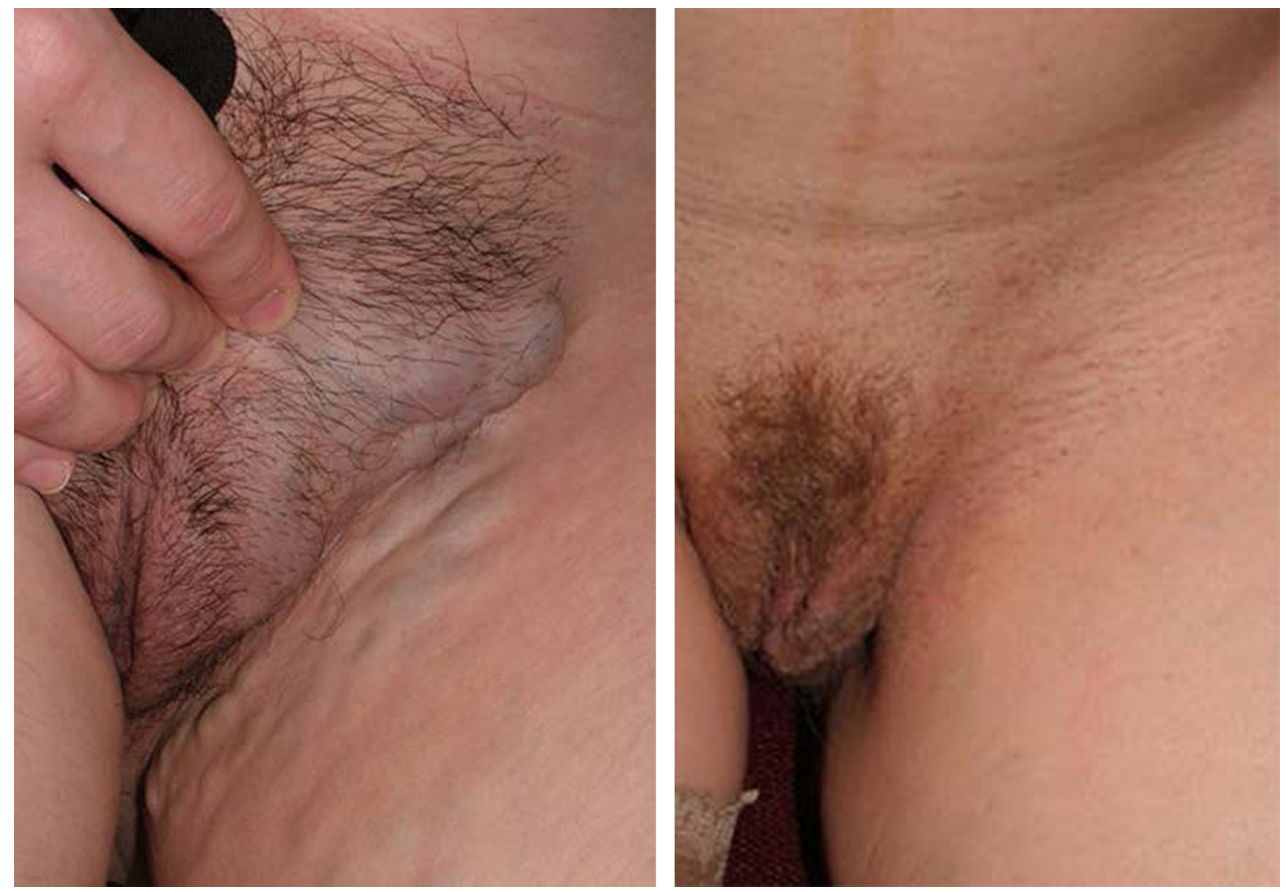

Figure 5 Appearance of vulvar varicose veins in pregnant women. 


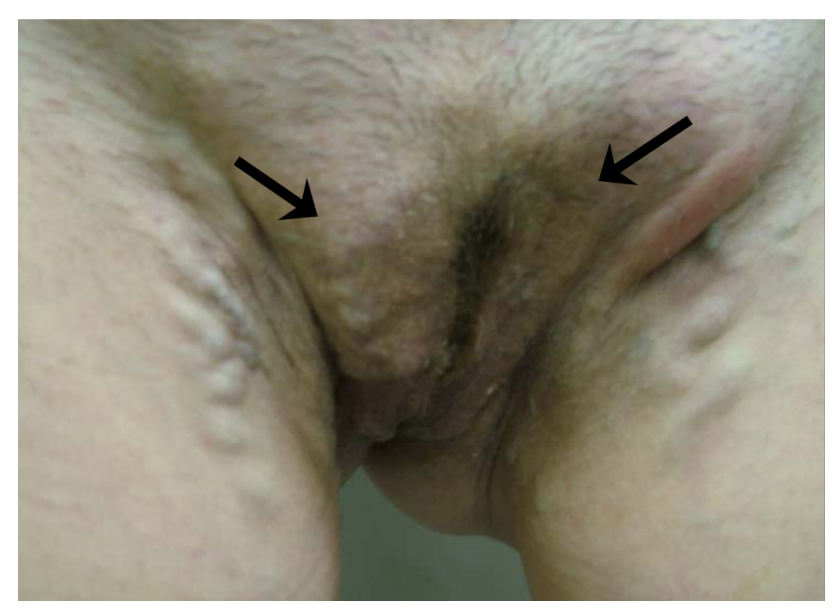

Figure 6 Thrombophlebitis of the vulvar veins. Hyperemia and edema in the area of thrombosed veins (arrow).

of the conducting veins of the pelvis, the inferior vena cava, or renal veins were identified.

A comparison of the clinical and instrumental data revealed that in patients with varicose transformation of the intrapelvic and vulvar veins, chronic pelvic pain occurred most often when valvular insufficiency of ovarian, uterine, or parametrial veins was present (ie, in $74 \%$ of patients with concomitant valvular insufficiency), but in only $10.5 \%$ of patients with isolated dilation of the pelvic venous plexuses. In addition, six women with dilation and abnormal reflux in the ovarian veins and intrapelvic venous plexuses had no signs of pelvic venous congestion, and four women, despite dilation of ovarian veins, had no associated valvular insufficiency or
Table I Duplex ultrasound results in patients with vulvar varicosities and varicose veins of the pelvis $(n=6 I)$

\begin{tabular}{lll}
\hline Vessel & $\begin{array}{l}\text { Diameter } \\
(\mathbf{m m})\end{array}$ & $\begin{array}{l}\text { Incidence of pathological } \\
\text { reflux (\%) }\end{array}$ \\
\hline Vulvar veins & $6.3 \pm 0.8$ & 100 \\
Uterine veins & $5.4 \pm 1.2$ & 89.5 \\
Parametrial veins & $7.6 \pm 0.7$ & 89.5 \\
Ovarian veins & $6.4 \pm 1.8$ & 37.7 \\
\hline
\end{tabular}

signs of pelvic venous congestion. This variation between the clinical and ultrasound data suggests extreme individuality in the clinical course of varicose veins of the pelvis, which is modified significantly with the formation of vulvar varicosities. The duplex-ultrasound results for pelvic veins in patients with vulvar varicosities and varicose veins of the pelvis are shown in Table 1. Seventeen patients with vulvar varicosities, clinical signs of pelvic venous congestion, and dilation and reflux in the ovarian veins who were scheduled for surgical intervention underwent MSCT or SOPP. Scan results from one of these patients are shown in Figure 7.

\section{Group 2}

Instrumental examination of pregnant women with vulvar varicosities was limited to duplex ultrasound of the veins of the perineum and lower extremities. This diagnostic test was required not only to verify the diagnosis but also to exclude latent thrombosis in the inferior vena cava in the presence of subjective symptoms. Dilation of the external pudendal vein and associated reflux, which represents an obvious
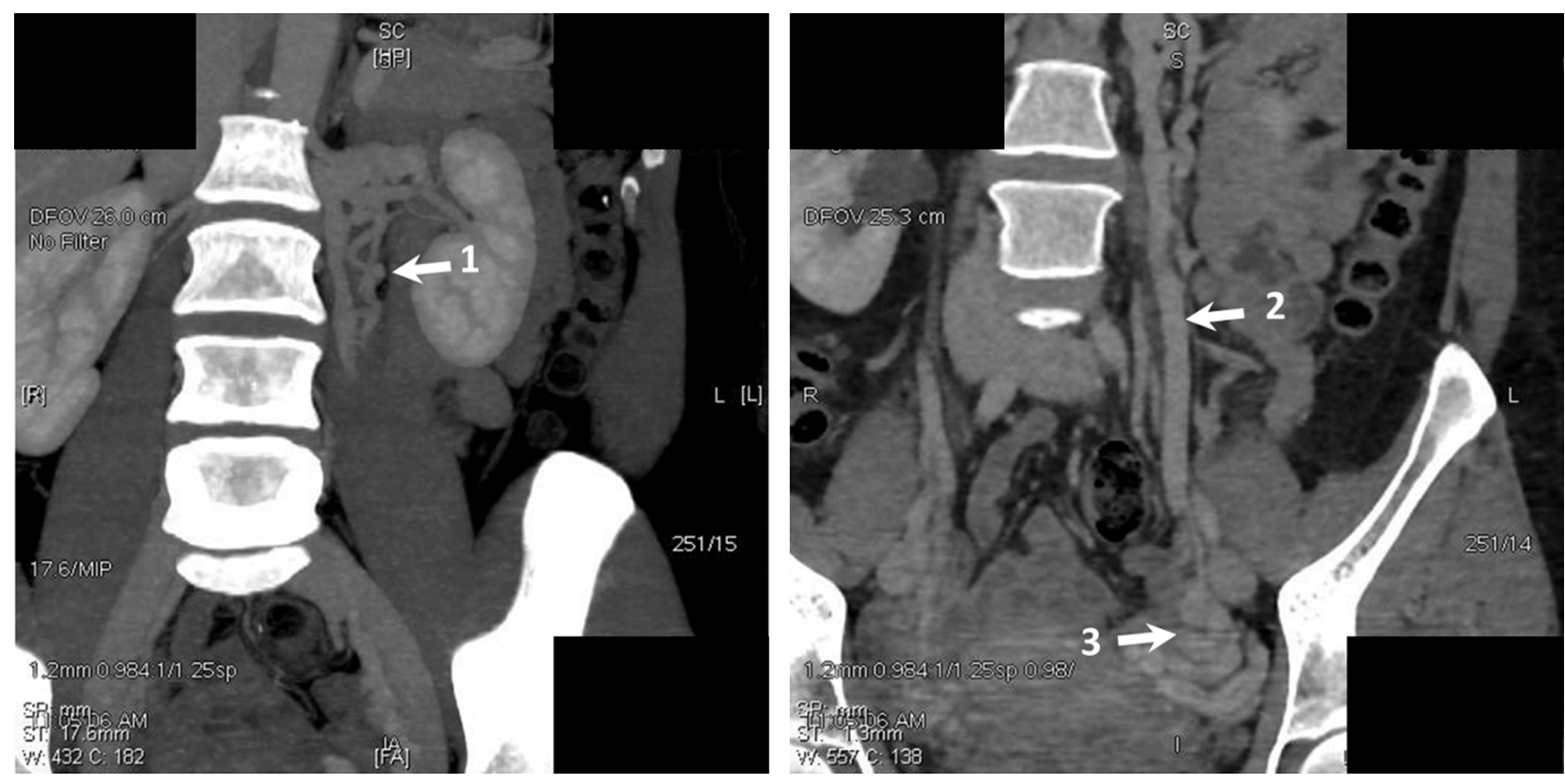

Figure 7 Computer tomography (CT) scans obtained in patient $\mathrm{K}$.

Notes: The tortuous left accessory ovarian vein (left) and the trunk of the left ovarian vein. The dilated grapelike plexuses and parametrial veins are visualized. I= additional left gonadal vein; $2=$ left gonadal vein; $3=$ the dilated grapelike plexuses and parametrial veins. 
reason for formation of vulvar varicosities, was found in $56 \%$ of patients. This rate was significantly higher than in group 1, where varicose transformation of the external pudendal vein was found in only $4.9 \%$ of patients. Figure 8 is from a patient at 24 weeks of pregnancy, and shows vulvar varicosity and venous nodes in the inguinal region at the projection of the saphenofemoral junction. Even at the clinical examination, these signs should raise an index of suspicion for valvular insufficiency at the orifice of the great saphenous vein and for blood reflux from the external pudendal vein into the vulvar vein, thus contributing to varicose transformation.

Saphenofemoral incompetence was found in 14 (35\%) pregnant women, of whom nine $(64 \%)$ had varicose syndrome in the lower extremities. The vulvar veins were significantly dilated $(1.1 \pm 0.54 \mathrm{~cm}$ in diameter $)$ compared with patients from group $1(6.3 \pm 0.8 \mathrm{~cm})$. In seven $(28 \%)$ women, the disorder was characterized by a combination of vulvar varicosities with varicose disease of the lower extremities and reflux in the saphenofemoral junction and estuarine tributaries. Dilation of the veins of the uterus and parametrium of varying degrees was observed in all patients followed up postpartum. In pregnant women, the results of

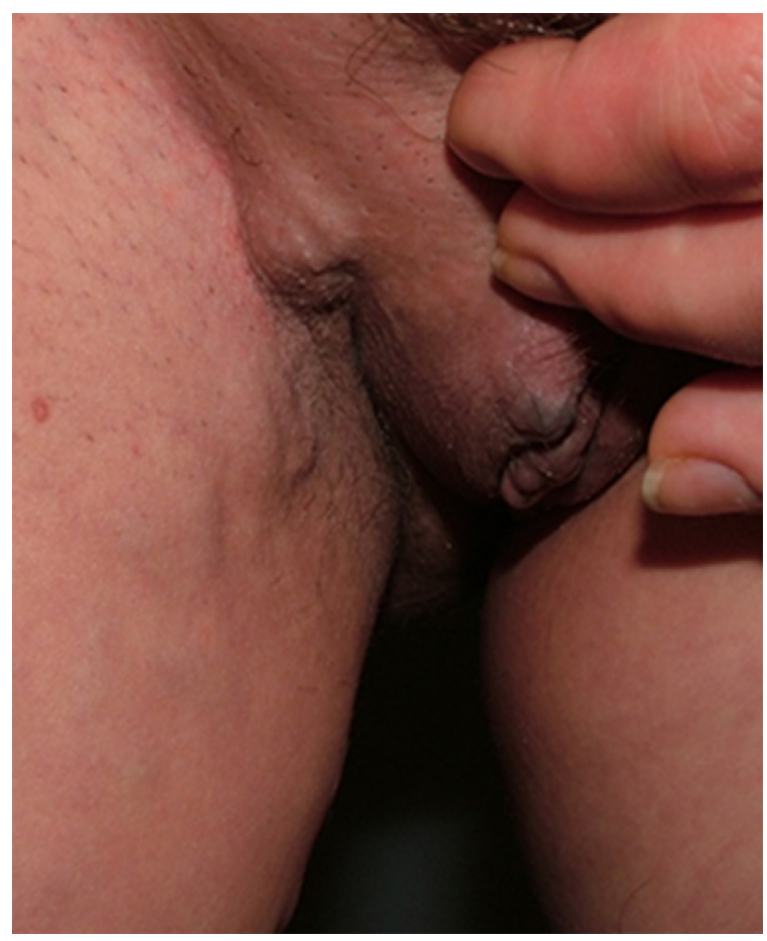

Figure 8 The appearance of vulvar and inguinal varices in a pregnant woman at 24 weeks' gestation.

Notes: The image shows vulvar varicosity and venous node in the inguinal region at the projection of the saphenofemoral junction. The varicose tributary of the great saphenous vein can be visualized in the upper third of the anteromedial side of the thigh. the duplex ultrasound were fully consistent with the clinical course of a chronic venous disorder: systemic incompetence of veins in most women, prevalence of symptoms at the level of the vulvar veins and superficial veins of the lower extremities, and significant dilation of the vulvar veins compared with group 1 patients with vulvar varicosities and varicose veins of the pelvis.

The course of vulvar varicosities in pregnant women is characterized by its complete disappearance in the postpartum period in some patients. Follow-up of 25 patients who developed vulvar varicosities during pregnancy for at least 1 year after childbirth revealed that dilated vulvar veins persisted in only $20 \%$ of patients. A reduction in vulvar varicosity was observed from the first days after birth, and most patients reported their complete disappearance within 2-8 months (5.8 \pm 1.04 months on average).

An association was found between the end of lactation period (or a reduction in breastfeeding) and the rate of vulvar varicosity disappearance: the shorter the lactation period, the earlier the varicose veins of the perineum disappeared, and vice versa. This once again indicates that hormonal changes play an important role in the development of varicose veins of the lower extremities, in the perineum, and in the small pelvis during pregnancy.

Transvaginal and transabdominal duplex ultrasound was performed in two patients who complained of pain in the lower abdomen lasting for 7 and 10 days at gestational ages of 28 and 30 weeks, respectively. Patients were examined by an obstetrician-gynecologist and underwent ultrasound of the pelvic organs and fetus. No obstetric pathology was identified. Ultrasound examination did not reveal any thrombotic lesions of the intrapelvic veins, and confirmed varicose veins of the uterus, parametrium, and vagina, with abnormal reflux flow. Of note, in some cases it is reasonable to perform duplex ultrasound of the veins of the vagina, in order to predict the risk of intrapartum hemorrhage. This is usually performed in pregnant women with large varicose veins of the vagina that are identified visually at the gynecological examination. There were no such patients in our study.

Duplex ultrasound confirmed the presence of vulvar vein thrombophlebitis in two women. These patients underwent ultrasound examination of the veins of the lower extremities. In one patient, the propagation of thrombosis to the external pudendal vein with ostial localization of thrombi was detected. In order to exclude latent thrombosis of the pelvic veins, this patient underwent ultrasonography, which showed no thrombi in the inferior vena cava, iliac veins, or their tributaries. 
Further instrumental examination of patients in group 2 was considered inappropriate, as pathological changes in the venous system of the inferior vena cava in most cases undergo regression in the postpartum period. Diagnostic and therapeutic problems related to pelvic vein abnormalities generally resolve after the end of the lactation period and restoration of a normal menstrual cycle.

\section{Treatment}

\section{Group I}

Surgical procedures in patients with vulvar varicosities and pelvic varicose veins were dependent on the clinical presentation and results of the instrumental examination. Studies performed at our clinic suggest that a clinical picture of pelvic venous congestion, dilation, and reflux in the ovarian veins is an absolute indication for surgery on ovarian veins, regardless of the presence or absence of vulvar varicosities. According to this principle, nine patients underwent extraperitoneal resection of the left ovarian vein, three patients extraperitoneal bilateral resection of ovarian veins, and five patients endovascular embolization using metal spirals. These interventions were followed by phlebectomy in the perineum.

In 32 patients with a painless form of vulvar varicosities and pelvic varicose veins, an isolated phlebectomy in the perineum was performed, regardless of the involvement of the ovarian veins in the pathological process. Preference was given to the removal of varicose veins via small incisions (up to $1 \mathrm{~cm}$ ) that allowed complete removal of conglomerates of veins of the labia majora and reliable hemostasis (Figure 9).

When varicose transformation of the veins of the labia minora was accompanied by hypertrophy, varicose veins were resected together with the labia minora (Figure 10). This procedure was performed in three patients. There were no complications after the surgery, and primary wound healing was observed in all patients. Emission CT of the pelvic veins using in vivo labeled red blood cells was performed both prior to and after surgery in 24 patients with vulvar varicosities and pelvic varicose veins. No occurrence or worsening of the specific signs of pelvic venous congestion was observed. There were no negative changes in the clinical symptoms of pelvic venous congestion either, if any of them presented in a patient prior to surgery. The follow-up period after surgery on perineum veins extended for 3-8 years, and during this period there were no recurrences of vulvar varicosities in any of the 32 patients.

Sclerotherapy of vulvar veins was performed as the primary treatment in 12 patients. Selection criteria for this procedure were the absence of an obvious connection between the dilated vulvar vein and the large tributary of the internal iliac vein (according to duplex-ultrasound results), and a venous diameter of no more than 5-6 $\mathrm{mm}$. It should be noted that, duplex ultrasound is not $100 \%$ accurate at tracking the route of a vein from the perineum into the small pelvis, but we refrained from sclerotherapy if we found vulvar vein drainage into the large intrapelvic branch. Contrast-enhanced MSCT would probably be of value in determining the intrapelvic junctions, but this issue requires further investigation. In all cases, liquid forms of sclerosing agents were used (Aethoxysklerol 1\% [Kreussler Pharma, Wiesbaden, Germany], Fibro-vein 0.5\% [STD Pharmaceutical Products, Hereford, UK]). Around 1.5-2 mL of sclerosing agent was injected into a vein of a patient in the supine position, followed by manual compression of the injection site for 5-7 minutes. Patients were advised to
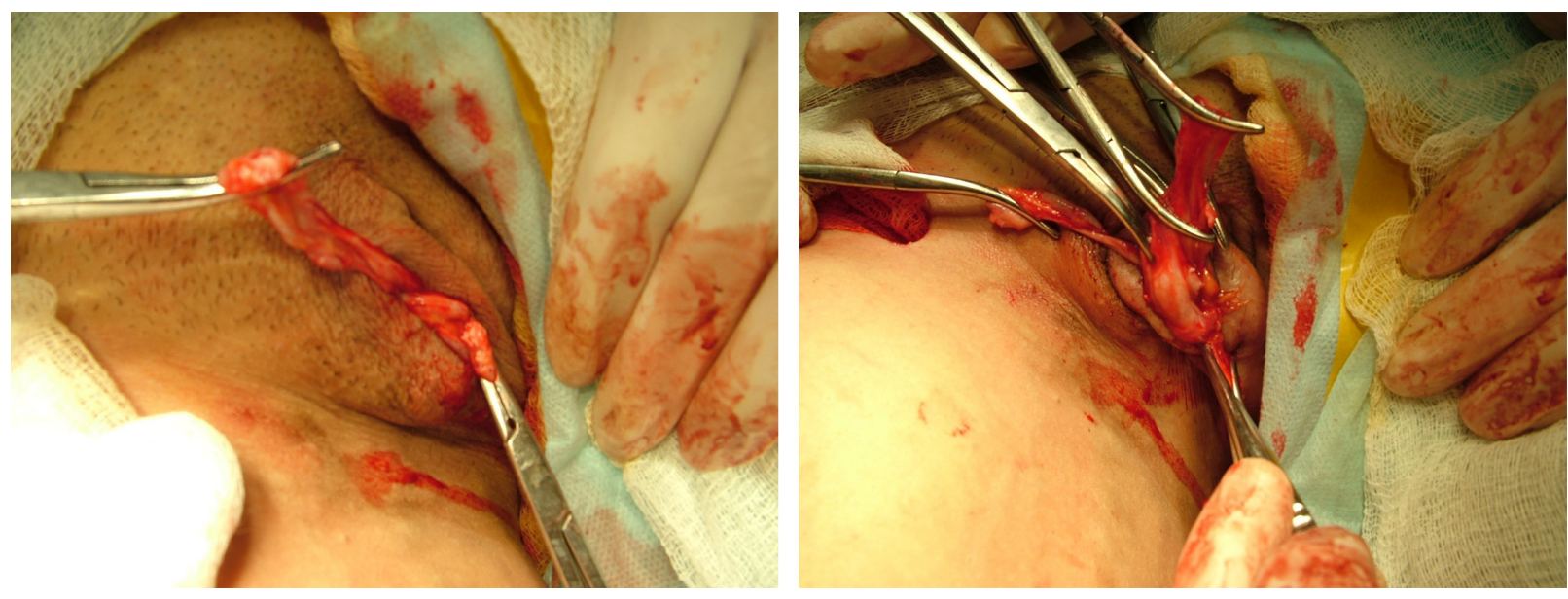

Figure 9 Stages of phlebectomy in the perineum. The large varicose vein of the right labia majora is mobilized. 

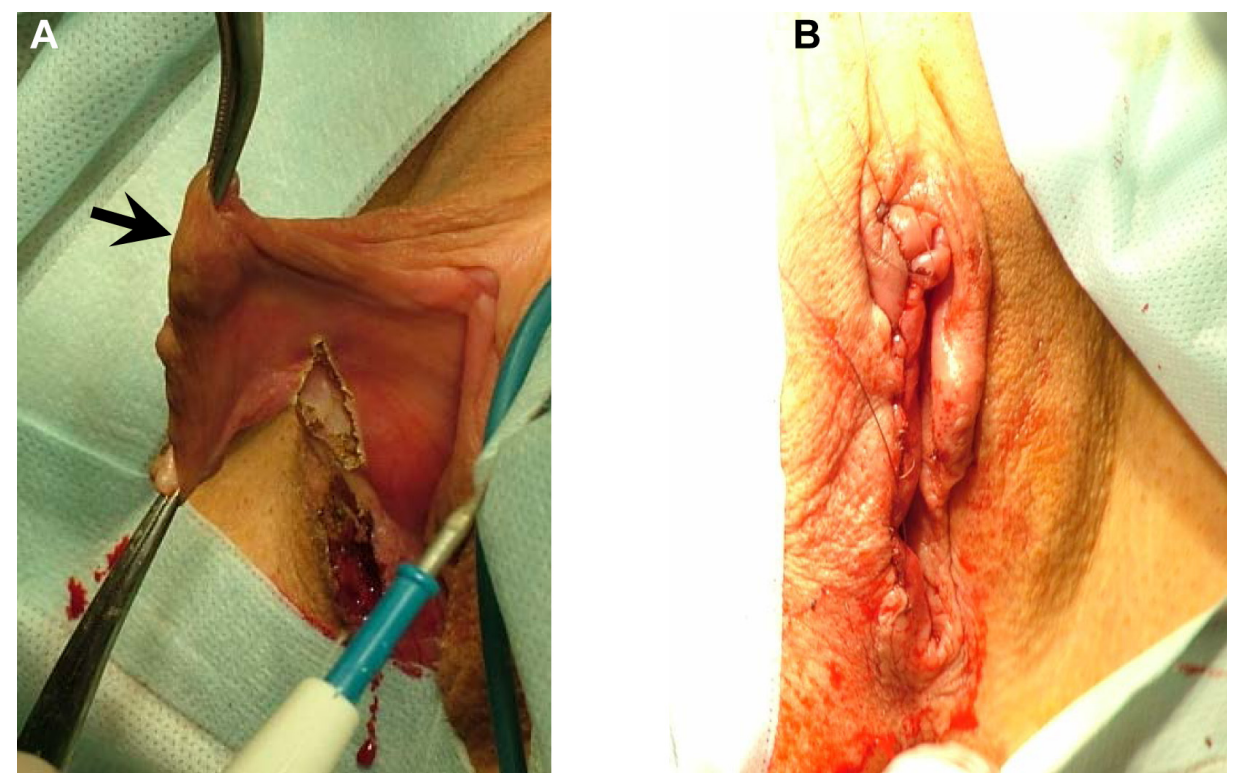

Figure 10 Resection and repair of the right labia minora. Photos illustrate the stages of the procedure. The arrows indicate varicose veins of the right labia minora. Notes: The right labia minora (A). The final type of operation (B).

wear tight elastic underwear with gauze or latex rollers in the area of the varicose veins for the next 7-10 days. In cases of persisting varicose veins, a repeat sclerotherapy session was performed after resolution of the phlebitis. One injection was sufficient in nine patients, while repeat injections were required in three patients. Figure 11 illustrates the improvement in the vulvar varicosity approximately 2 weeks after sclerotherapy.

No complications after sclerotherapy (skin changes, thrombosis of pelvic veins, pulmonary embolism, or allergic reactions) were observed. During the first year of follow-up, a consistent therapeutic and cosmetic effect (no vulvar varicose veins, pain, or discomfort in the perineum, nor swelling of the vulvar lips) was noted in ten patients. Vulvar varicosity relapsed in two patients at 2 and 3 months after the procedure, respectively, most likely because these patients became pregnant soon after the sclerotherapy.

Conservative treatment formed an essential part of both surgical treatment and sclerotherapy. As part of the preoperative preparation, 38 patients with vulvar varicosities were randomly chosen to complete a treatment course with the venotonic drug micronized purified flavonoid fraction (MPFF) at a dose of $1,000 \mathrm{mg}$ daily for 2 months prior to the surgical procedure. Treatment was associated with a significant reduction in symptoms, such as pain, heaviness, and discomfort in the perineum and swelling of the labia majora (Figure 12).

\section{Group 2}

Venoactive drugs formed the cornerstone of treatment for vulvar varicosities during pregnancy. In the majority of
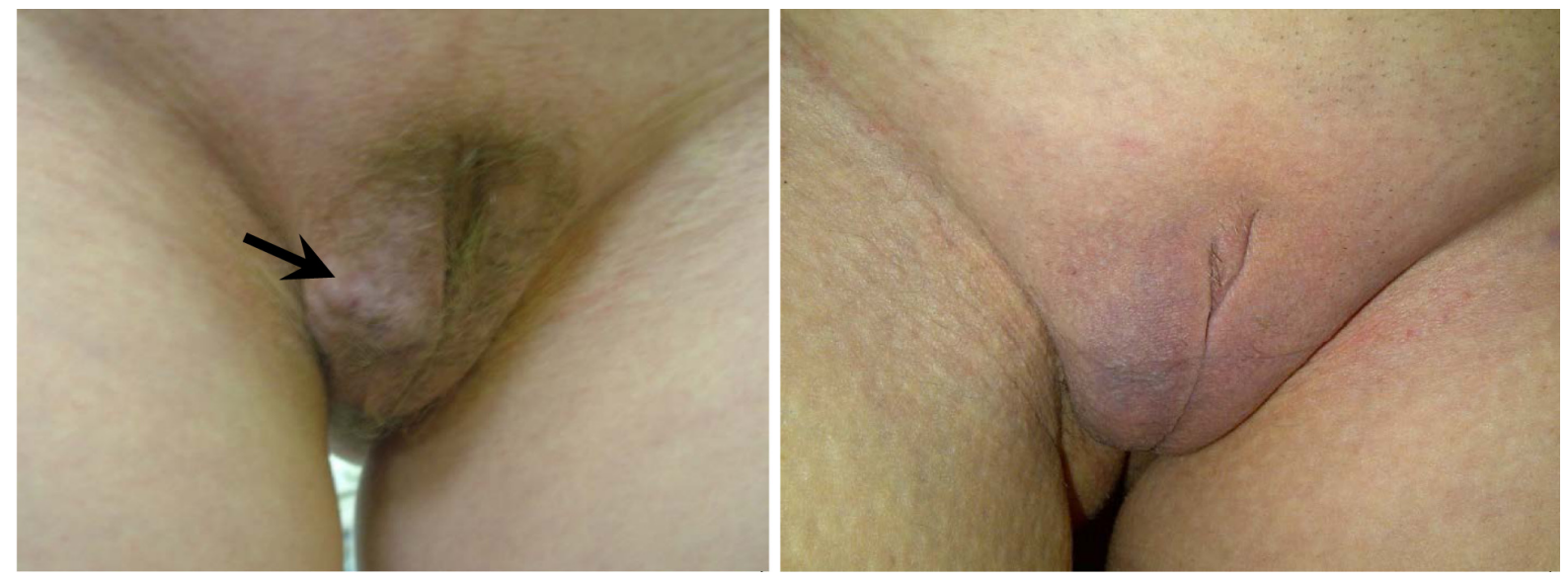

Figure II The appearance of vulvar varicose veins before (left) and after (right) sclerotherapy of the vulvar varicosity. The arrow indicates the veins of the right labia majora. 


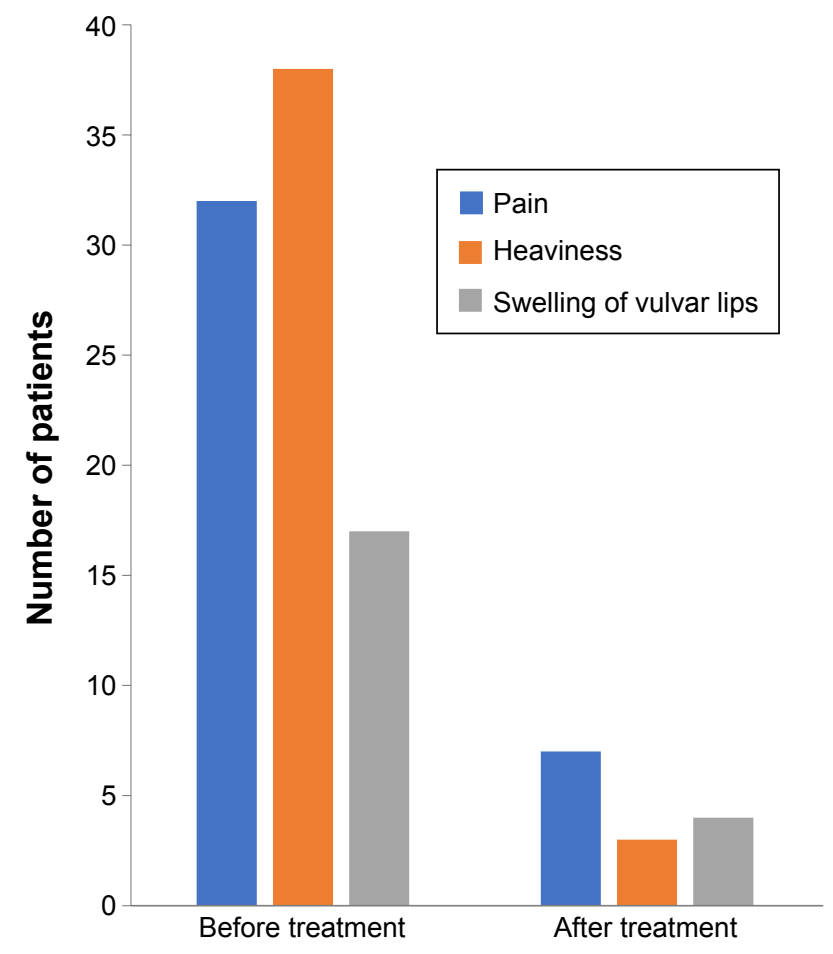

Figure 12 Changes in the symptoms of vulvar varicosity during treatment with micronized purified flavonoid treatment.

women, dilation of the pudendal veins occurs during the second and third trimesters of pregnancy, allowing the use of MPFF (Detralex), which is approved for the treatment of chronic venous disease from the second trimester of pregnancy. The use of MPFF did not lead to the complete cessation of symptoms associated with vulvar varicosities, but reduced their severity. In cases of skin itching and skin maceration in the region of vulvar varicose veins, $\mathrm{H}_{1}$ histamine-receptor blockers and zinc oxide paste were recommended to relieve symptoms. For compression treatment, patients were advised to wear tight elastic underwear with gauze or special latex rollers. This helped reduce such symptoms as heaviness and swelling of the vulvar lips. In Russia, specialized compression hosiery for women with vulvar varicose veins is not available.

Surgical treatment of vulvar varicosities during pregnancy is indicated only in cases of complications. In our study, only one patient with thrombophlebitis of the vulvar veins and ostial thrombophlebitis of the left external pudendal vein underwent an urgent surgical crossectomy. The development of local thrombophlebitis of the vulvar veins is an indication for anticoagulant therapy with modern low-molecular-weight heparin at intermediate doses. Anticoagulant prophylaxis of venous thromboembolic events in pregnant women with vulvar varicosities was carried out in cases of thrombophlebitis of the vulvar veins or when there was a combination of vulvar varicosities with pronounced varicose veins of the lower extremities. In our view, a preventive dose of low-molecular-weight heparin does not lead to significant changes in hemostasis, but very likely prevents venous thrombosis and pulmonary embolism during pregnancy. We used such an approach in 23 patients in group 2. No complications of preventive treatment with heparin were observed during the follow-up period.

An important aspect of vulvar varicose veins in pregnancy is the mode of delivery. Obstetricians often consider a conglomerate of varicose veins on the vulvar lips an indication for cesarean section. Our experience shows that vulvar varicose veins are not a contraindication to natural delivery, as the perioperative risks for cesarean section outweigh the risks of bleeding from varicose veins of the vulvar lips. The latter is extremely rare, and is easily resolved by ligating the bleeding vessel. Significantly dilated veins of the vagina (vaginal varicosity) can be considered an indirect indication for cesarean section. Visual detection of large $(1 \mathrm{~cm}$ or more) venous nodes on the vaginal wall, confirmed by the results of ultrasonography, should alert both obstetrician and phlebologist or vascular surgeon when choosing the mode of delivery. However, in such situations, it is necessary to consider not only the vaginal varicose veins themselves but also other factors that may contribute to the occurrence of venous bleeding during delivery, primarily obstetric problems. Such an approach helps avoid unnecessary operations and minimizes the risk of bleeding from varicose veins during the delivery period.

\section{Discussion}

The true prevalence of vulvar varicosities is difficult to establish, as women are often reluctant to draw attention to them, particularly if they do not cause any significant discomfort. In the current study, vulvar varicosities were found to accompany varicose veins of the pelvis in $32 \%$ of cases, and occurred most often in women with a history of two or more full-term pregnancies (91\%). In a surgical clinic, it is not possible to assess the incidence of vulvar varicosities in pregnant women, as gynecologists generally refer all patients with this condition for consultation. Study of the epidemiology of vulvar varicosities thus requires collaboration between obstetrician-gynecologists and phlebologists.

We evaluated two groups of women: nonpregnant patients with varicose veins of the pelvis and enlarged vulvar veins (group 1), and pregnant women with vulvar varicosities (group 2). In both groups, bilateral dilation of the veins of 
the pudendum was more frequent than ipsilateral dilation, indicating that significant and sustainable pelviperineal blood reflux occurs during pregnancy, which persists during the postpartum period and leads to the formation of pelvic varicose veins. In group 1, dilation of ovarian, uterine, and parametrial veins was not always accompanied by abnormal reflux, indicating that blood-flow disturbances are not always present.

In group 1 women, each subsequent pregnancy had been accompanied by an earlier appearance and greater dilation of the vulvar veins. Another observation from this group was that women with vulvar varicosities and pelvic varicose veins had a lower incidence of chronic pelvic pain (31\%) than women with pelvic varicose veins and no vulvar varicosities (70\%). In our opinion, this may be due to several factors. First, the connection between the vulvar and intrapelvic veins can result in a significant reduction in pelvic pain, due to shunting of blood from the pelvic venous plexuses to the veins of the perineum. Second, based on the concept of venous nociceptive pain, it can be assumed that in some patients, there are more nociceptors in the superficial veins of the lower extremities and perineum than in the pelvic veins. Third, long-lasting pelvic venous congestion may lead to the death of pain receptors in the veins of the pelvis and consequently the absence of chronic pelvic pain or its disappearance in patients with pelvic varicose veins. Fourth, the number and localization of estrogen and progesterone receptors in the venous wall may vary between individuals, which could explain the selective lesions of the veins of pelvis and perineum during pregnancy, as well as the prevalence of vulvar varicosity over intrapelvic varicosity, and thus absence of the signs of pelvic venous congestion. Finally, it is important to take into account a patient's individual response to pain. Minor pain in one patient may cause a violent emotional reaction and an abundance of complaints, while in another the presence of chronic pelvic pain may not have a significant effect on quality of life.

Women who underwent surgery on the veins of the perineum were followed up for 3-8 years, and during this period there were no recurrences of vulvar varicosities in any of the 32 patients. This suggests that removal of vulvar veins does not lead to a deterioration of venous drainage from the pelvic organs, and achieves a long-term recurrence-free postoperative course. The procedure is certainly indicated in patients with severe vulvar varicosities, varicose veins of more than 5-6 mm in diameter, and suspected or clearly established communication with a large intrapelvic vein.

In patients with vulvar varicosities and a painless form of pelvic varicose veins, there is a need for the development and introduction into clinical practice of hybrid surgical procedures aimed at the elimination of vulvar varicosities and reflux in the tributaries of the internal iliac vein, ie, phlebectomy in the perineum and endovascular embolization of the obturator and internal pudendal veins, which play a major role in the formation of vulvar varicosities. Several studies have demonstrated success with the use of intravenous embolization for the treatment of vulvar varicosities. ${ }^{6-8}$ In one study, venous reflux was found to be the cause of vulvar and/or posteromedial thigh varicosities in 23 of 25 women, and left ovarian venous reflux was responsible for $60 \%$ of all cases. Embolization using multiple coils was successful in 18 of 23 patients (78.3\%). ${ }^{6}$ In another study, coil embolization of the ovarian vein resulted in the disappearance of vulval varices in 21 of $24(88 \%)$ patients. ${ }^{7}$

Vulvar varicosities are only a symptom of pelvic varicose veins, and so their treatment should be combined with the elimination of the cause of the disease, namely correction of pelviperineal reflux if it exists according to data from pelvic venography or MSCT. Embolization of the dilated and incompetent ovarian veins that allow retrograde blood flow and contribute to venous congestion in the uterus and ovaries can be considered a measure for preventing the development of vulvar varicosities in patients with pelvic varicose veins.

Out of the 12 women who underwent sclerotherapy as the primary treatment, there was a reoccurrence of vulvar varicosities in two patients at 2 and 3 months after the procedure, respectively, most likely due to these women becoming pregnant soon after the sclerotherapy procedure. A significant and sustained increase in progesterone concentrations during the early stages of pregnancy results in a reduction in venous tone and worsens pelviperineal blood reflux. On the other hand, the reoccurrence of vulvar varices could have been caused by the lack of adequate compression of the perineum after the procedure, as elastic hosiery that produces a compression effect on the vulvar and perineal veins is not currently available in Russia.

Sclerotherapy should continue to be recognized as an effective method for the reduction of vulvar varicosity symptoms. Another advantage is that it can be performed in an outpatient setting without the need for specialized methods of anesthesia. However, to achieve a positive result, it is necessary to select patients carefully for this type of treatment, in order to avoid serious complications, such as thrombosis of the pelvic veins and pulmonary embolism.

Research has suggested that only a minority of incompetent ovarian veins will present with clinically detectable lowerlimb venous insufficiency. ${ }^{9}$ A comprehensive assessment of the state of the vulvar and intrapelvic veins is necessary to 
define appropriate treatment strategies in patients with vulvar varicosities that will reduce symptoms and improve quality of life. In this respect, contrast-enhanced CT of the pelvic organs may be of value to document the connection between the vulvar and intrapelvic veins, and to perform a reference assessment of the dilation of these vessels.

Conservative treatment with the venoactive agent MPFF was valuable as both a systemic treatment for chronic venous disease and rehabilitation of patients after surgery. Persisting varicose transformation of the veins of the uterus and parametrium, despite the absence of the signs of pelvic venous congestion, is an indication for treatment with venoactive drugs. These provide a beneficial effect on the vulvar and intrapelvic veins, and in our opinion reduce existing pelviperineal blood reflux, thereby serving as a means to prevent the development of pelvic congestion. Previous research has shown that when MPFF is started at the time of surgery and continued for 4 weeks it improves the results of varicosevein intravenous procedures and hemorrhoidal procedures by reducing postoperative symptoms and the need for analgesics and anti-inflammatory drugs. ${ }^{10}$ In an animal study, it was shown to reduce the side effects of sclerotherapy by minimizing the inflammatory response within the surrounding tissues when treatment was started 7 days before sclerotherapy. ${ }^{11}$

Pregnancy is a major factor in the development of vulvar varicosities, but it is difficult to suggest preventive methods aimed at reducing the risk of their formation, as the prescription and implementation of various therapeutic measures

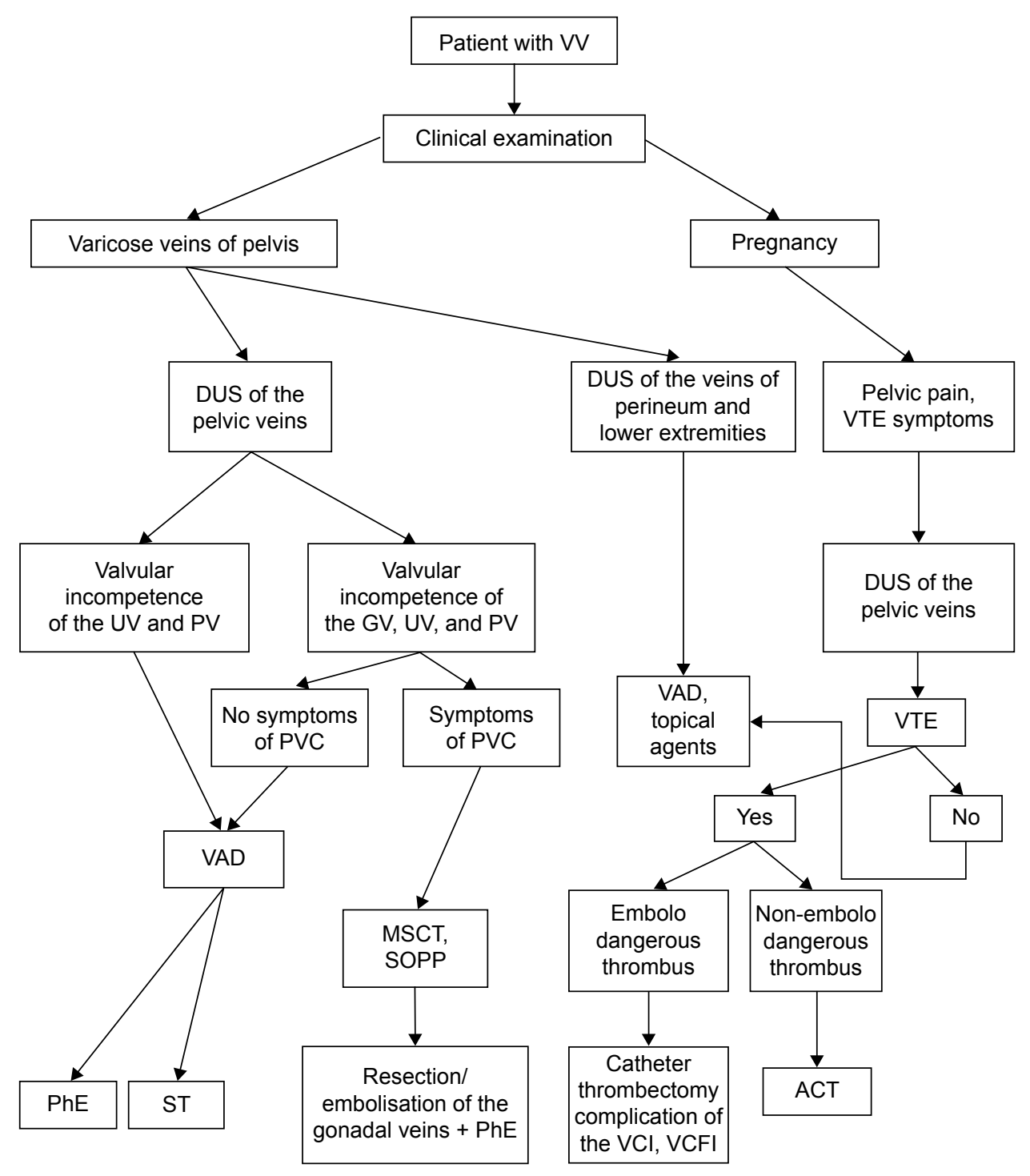

Figure 13 Diagnostic and treatment algorithm for vulvar varicosities in various clinical situations.

Abbreviations: VV, vulvar varicosity; DUS, duplex ultrasound; VTE, venous thromboembolic event; UV, uterine vein; PV, parametrial vein; GV, gonadal (ovarian) vein; PVC, pelvic venous congestion; VAD, venoactive drug; MSCT, multislice computed tomography; SOPP, selective ovariography with pelvic phlebography; PhE, phlebectomy; $\mathrm{ST}$, sclerotherapy; $\mathrm{VCl}$, vena cava inferior; VCFI, VC-filter implantation; ACT, anticoagulant therapy. 
is limited. However, compliance with dietary modification and simple recommendations for the proper organization of patient work and rest, excluding prolonged periods of standing and heavy physical exertion, can be considered the simplest preventive measures. Therapeutic exercises aimed at accelerating venous outflow from the legs and small pelvis organs can also reduce venous congestion. Symptoms of functional phlebopathy and the presence of varicose veins of the pelvis and/or lower extremities before pregnancy are indications for the use of venoactive drugs and compression therapy.

One of the most effective methods of prevention is timely interventions on the ovarian veins (resection, embolization). This will reduce the effects of pelvic venous congestion by eliminating vertical reflux of blood through the ovarian veins. This in turn reduces the load on the tributaries of the internal iliac veins, which are in most cases the sources of vulvar varicosity. Given that pelvic varicose veins are a disease that affects women of childbearing age, it is reasonable to consider surgical interventions on the ovarian veins in asymptomatic patients, in whom the long-term existence of venous congestion of the pelvic organs may affect reproductive function. It is possible that this will reduce the risk of venous thromboembolic events in pregnancy and childbirth and minimize the risk of developing pelvic venous congestion and vulvar varicose veins.

At present, surgical treatment of symptomatic forms of pelvic varicose veins predominates, and is limited to the ovarian veins. However, in some patients pelvic venous congestion develops without any impairment of these veins, but exclusively due to the development of valvular insufficiency in the internal iliac vein and its tributaries. The development of techniques for endovascular intervention at this level will enhance the ability of surgeons to treat both pelvic varicose veins and vulvar varicosities. These procedures will be of value in patients with isolated dilation of the pelvic venous plexuses in whom conservative treatment has failed, and who have progression of pelvic venous congestion symptoms, and vulvar varicosities in particular.

It should also be remembered that vulvar varicosities can be caused by reflux in the external pudendal vein and saphenofemoral incompetence associated with varicose disease of the lower extremities. Timely surgical treatment of this disease should thus also be considered a measure of vulvar varicosity prevention.

\section{Conclusion}

Vulvar varicosities are a common venous disorder prevalent among women with varicose veins of the pelvis and lower extremities and in pregnant women. In most cases, vulvar varicosities can be diagnosed at clinical examination, and do not require any special investigation methods. Their diagnosis requires an assessment of the state of the intrapelvic veins, and in cases of pregnancy further observation and examination in the postpartum period. Treatment varies from purely conservative measures during pregnancy to various surgical procedures on the ovarian and vulvar veins. A diagnostic and treatment algorithm for vulvar varicosities in various clinical situations is presented in Figure 13. An individualized approach to diagnostic methods and treatment for this disorder can significantly improve the quality of care of patients with chronic venous diseases.

\section{Acknowledgment}

Editorial assistance was provided by Jenny Grice, and funded by Servier International.

\section{Disclosure}

The author reports no conflicts of interest in this work.

\section{References}

1. Saveliev VS, Pokrovsky AV, Zatyevakhin II, Kirienko AI. Rossiiskie klinicheskie rekomendatsii po diagnostike i lecheniyu khronicheskikh zabolevanii ven. Flebologia. 2013;7:18-20.

2. Fassiadis N. Treatment for pelvic congestion syndrome causing pelvic and vulvar varices. Int Angiol. 2006;25:1-3.

3. Bell D, Kane PB, Liang S, Conway C, Tornos C. Vulvar varices: an uncommon entity in surgical pathology. Int $J$ Gynecol Pathol. 2007;26:99-101.

4. Thomas ML, Fletcher EW, Andress MR, Cockett FB. The venous connections of vulval varices. Clin Radiol. 1967;18:313-317.

5. Ignacio EA, Dua R, Sarin S, et al. Pelvic congestion syndrome: diagnosis and treatment. Semin Intervent Radiol. 2008;25:361-368.

6. Ashour MA, Soliman HE, Khougeer GA. Role of descending venography and endovenous embolization in treatment of females with lower extremity varicose veins, vulvar and posterior thigh varices. Saudi Med $J$. 2007;28:206-212.

7. Castenmiller PH, de Leur K, de Jong TE, van der Laan L. Clinical results after coil embolization of the ovarian vein in patients with primary and recurrent lower-limb varices with respect to vulval varices. Phlebology. 2013;28:234-238.

8. Aslam MI, Flexer SM, Figueiredo R, Ashour HY, Bhattacharya V. Successful embolization of vulval varices arising from the external pudendal vein. J Surg Case Rep. 2014;2014:rjt129.

9. Sutaria R, Subramanian A, Burns B, Hafez H. Prevalence and management of ovarian venous insufficiency in the presence of leg venous insufficiency. Phlebology. 2007;22:29-33.

10. Astashov V, Timchenko D. Benefits of micronized purified flavonoid fraction in the reduction of symptoms after operation for hemorrhoidal disease. Phlebolymphology. 2013;21:95-99.

11. de Souza MD, Cyrino FZ, Mayall MR, et al. Beneficial effects of the micronized purified flavonoid fraction (MPFF, Daflon ${ }^{\circledR} 500 \mathrm{mg}$ ) on microvascular damage elicited by sclerotherapy. Phlebology. 2016;31:50-56. 
International Journal of Women's Health

Dovepress

\section{Publish your work in this journal}

The International Journal of Women's Health is an international, peerreviewed open-access journal publishing original research, reports, editorials, reviews and commentaries on all aspects of women's healthcare including gynecology, obstetrics, and breast cancer. The manuscript management system is completely online and includes

Submit your manuscript here: http://www.dovepress.com/international-journal-of-womens-health-journa a very quick and fair peer-review system, which is all easy to use. Visit http://www.dovepress.com/testimonials.php to read real quotes from published authors. 